\title{
Water extract of Cnidii Rhizoma suppresses RANKL-induced osteoclastogenesis in RAW 264.7 cell by inhibiting NFATc1/c-Fos signaling and prevents ovariectomized bone loss in SD-rat
}

\author{
Ka-Yeon Lee ${ }^{1 \dagger}$, Jae-Hyun Kim ${ }^{1 \dagger}$, Eun-Young Kim', Mijung Yeom², Hyuk-Sang Jung ${ }^{1}$ and Youngjoo Sohn ${ }^{1 *}$
}

\begin{abstract}
Background: Cnidii Rhizoma is the dried root stem of Cnidium officinale Makino. Cnidii Rhizoma (CR) has been used to treat menstrual irregularity, menstrual pain, and menopause in Korea. However, the effects and mechanisms of CR on RANKL-induced osteoclastogenesis pathway remain to be elucidated. In this study, we investigated the effects of CR on the inhibition of bone resorption of osteoclast and its mechanism RANK signaling pathway.

Methods: The anti-osteoclastogenesis of water extract of CR was measured using RAW 264.7 cell. Tartrateresistant acid phosphatase (TRAP) assay, pit assay, reverse transcription polymerase chain reaction (RT-PCR) and western blot were performed. Moreover, the effects of $C R$ were determined with an in vivo model using ovariectomized $(\mathrm{OVX})$ rats.

Results: CR extract suppressed osteoclastogenesis, its activity and bone resorption activity through decreasing gene of osteoclast-related such as nuclear factor of activated T-cells, cytoplasmic 1 (NFATc1), c-Fos, etc. Moreover, CR extract prevented the bone loss in OVX rats.

Conclusion: These results show that CR has a positive effect on menopausal osteoporosis by suppressing osteoclastogenesis.
\end{abstract}

Keywords: Cnidii Rhizoma, Osteoclast, NFATc1, c-Fos, Ovariectomized-rat

\section{Background}

Osteoporosis is a critical diseases characterized by bone loss and impaired bone quality that can lead to an increased risk of fracture $[1,2]$. In normal skeleton, bone remodeling occurs through bone resorption by osteoclasts and the synthesis of bone by osteoblasts. An imbalance in these processes can cause osteoporosis [3-5]. Osteoclasts are multinucleated giant cells that can resorb bone matrix. Osteoclasts can differentiate for many reasons, one of which is a deficiency of a sexual hormone

\footnotetext{
* Correspondence: youngjoos@khu.ac.kr

${ }^{\dagger}$ Ka-Yeon Lee and Jae-Hyun Kim contributed equally to this work.

${ }^{1}$ Department of Anatomy, College of Korean Medicine, Kyung Hee University, Seoul 02447, Republic of Korea

Full list of author information is available at the end of the article
}

such as estrogen, which leads to menopause, increased bone resorption activity by osteoclasts, and the increased formation of osteoclasts, and these factors have key roles in bone loss $[6,7]$. Therefore, osteoporosis can be treated by suppressing the activation of osteoclasts.

Osteoclasts are generated by the fusion of a monocyte and macrophage derived from osteoclast precursors induced by osteoclast cytokines, such as the receptor activator of the nuclear factor kappa B (NF-kB) ligand (RANKL). There are many experimental methods for osteoclastogenesis, such as using RAW 264.7 cells and BMM cells derived from mice [8, 9]. Among these methods, the use of the RAW 264.7 murine cell line has been demonstrated to be a significant tool for in vitro researches of osteoclastogenesis and its activity [10]. 
RANKL can conjugate to its receptor RANK which is located on the RAW 264.7 cell surface [11]. This conjugation leads to the expression of tumor necrosis factor receptor-associated factor 6 (TRAF6). This results in the activation of downstream signaling cascades including the nuclear factor of activated T-cells, cytoplasmic 1 (NFATc1) and c-Fos. Both transcription factors have critical and specific roles in osteoclastogenesis [12]. Moreover, NFATc1 can directly control osteoclast specific genes [5, 13-15].

Cnidii Rhizoma (CR) is the dried root stem of Cnidii officinale Makino, which is called "Chunkung" in Korea [16]. In oriental medicine, $C R$ has been used to treat menstrual irregularity, menstrual pain, and menopause for woman $[17,18]$. In previous study, CR has been reported to have various biological activities such as angiogenesis [19], anti-cancer [20,21], anti-oxidant [22] and anti-inflammatory effect [23]. Also, various studies have shown that anti-inflammatory and anti-oxidant effects are associated with osteoclast inhibition [24-26]. Recently, Ligusticum chuanxiong which is observed that the same chemical type of compound constitutes the main component of CR [27] has been shown to be effective in osteoblast activity [28]. Inhibition of osteoclast differentiation is more important than promoting osteoblast differentiation on postmenopausal osteoporosis [5]. However, studies on the effect of CR on osteoclasts have not yet been investigated. In typical anti-resorptive medicines for postmenopausal osteoporosis (bisphosphonate and hormonal therapy etc.), there are several side effects such as breast and uterine cancer, vascular disease $[4,29]$. In consideration of recent studies, we expected that if $\mathrm{CR}$, which is used for gynecological diseases, has effect of the inhibition for osteoclast differentiation and bone absorption in ovariectomized (OVX) osteoporosis model, it could be useful in the treatment of menopausal symptoms including postmenopausal osteoporosis.

In this study, we focused on determining whether water extract of $\mathrm{CR}$ suppressed osteoclastogenesis and the bone resorption activity by inhibiting RANKL induced NFATc1, c-Fos, and the RANK signaling pathway in RAW 264.7 cells. In addition, we carried out the alleviation effect in OVX rat's bone loss.

\section{Methods}

\section{Preparation of CR extracts}

$\mathrm{CR}$ was authenticated by Professor Yungmin $\mathrm{Bu}$ at the Herbology Laboratory, College of Korean Medicine, Kyung Hee University and purchased from Kyung Hee University Medical Center. The extract was prepared by decocting $300 \mathrm{~g}$ of the dried herb with $3 \mathrm{~L}$ of boiling distilled water for $2 \mathrm{~h}$ and then filtering it using filter paper. The extract was collected in a rotary evaporator and lyophilized, which yielded $81 \mathrm{~g}$ of dried powder (yield ratio $27 \%$ ), and stored at $-20^{\circ} \mathrm{C}$ until use. A voucher specimen of the plant material used in this study has been deposited in the Department of anatomy herbarium [KHU-ANA-A061].

\section{Analysis of CR extract with HPLC}

Standard stock solutions $(1000 \mu \mathrm{g} / \mathrm{ml})$ of chlorogenic acid (primary pharmaceutical reference standard, SigmaAldrich, Saint-Louis, MI, USA) were prepared in methanol. A Waters 2695 system equipped with a Waters 2487 Dual $\lambda$ absorbance detector was used for the analysis of both chlorogenic acid from CR and chlorogenic acid as the standard. The separation was carried out on an Xbridge-C18 $(250 \mathrm{~mm} \times 4.6 \mathrm{~mm}, 5 \mu \mathrm{m})$ with a C18 guard column. The binary mobile phase consisted of solvent $\mathrm{A}$, methanol, and solvent $\mathrm{B}$, water containing $1 \%$ acetic acid. All the solvents were filtered through a $0.45 \mu \mathrm{m}$ filter prior to use. The elution conditions were $0-30 \mathrm{~min}$. of $15 \% \mathrm{~A}$ and $85 \% \mathrm{~B}$ at a flow rate of $1.0 \mathrm{ml} / \mathrm{min}$ with an injection volume of $10 \mu \mathrm{l}$. Chlorogenic acid was detected at $350 \mathrm{~nm}$.

\section{Cell culture of the osteoclast precursor cells and cell viability}

RAW 264.7 cells (Korea cell line bank, Seoul, Korea), a cell line derived from murine macrophage cells, were maintained in Dulbecco's medium Modified Eagle (DMEM) supplemented with $10 \%$ fetal bovine serum (FBS) and antibiotics (1\% penicillin/streptomycin) at $37^{\circ} \mathrm{C}$ in an atmosphere containing $5 \% \mathrm{CO}_{2}$ that $95 \%$ humidity. Cell viability of RAW 264.7 cells for CR was determined using MTS solution (Promega, Madison, WI).

\section{TRAP assay and bone resorption assay}

For differentiation, RAW 264.7 cells were treated with RANKL $(100 \mathrm{ng} / \mathrm{ml})$ and CR for 5 days. For the TRAP staining, mature osteoclasts were washed with DPBS (Gibco, Gaithersburg, MD, USA), the differentiated RAW 264.7 cells were stained using the TRAP staining kit (Sigma Aldrich, Saint Louis, MI, USA) according to the manufacturer's protocol. TRAP-staining positive differentiated RAW 264.7 cells were counted under a microscope (Olympus, Tokyo, Japan). Measuring of the TRAP activity was performed as previously described [9]. RAW 264.7 cells were cultured in the osteo assay strip well plate (Corning Incorporated, New York, NY, USA) with RANKL and CR. The pit of the plate was captured under an inverted microscope.

\section{Western blot analysis}

The cells were lysed in lysis buffer $(50 \mathrm{mM}$ Tris. Cl, 150 $\mathrm{mM} \mathrm{NaCl}, 1 \%$ NP-40, 0.5\% Na.deoxycholate, 0.1\% SDS, and a protease inhibitor cocktail, phosphatase inhibitor cocktail). The equal amounts of proteins were separated 
by SDS-PAGE and transferred membrane (Whatman Protran, Dassel, Germany). The membrane was blocked and then incubated with primary antibodies (1:1000) such as NFATc1 (BD Pharmingen San Diego, CA, USA), c-Fos and Actin (Santa Cruz Biotechnology, Santa Cruz, CA, USA) followed by secondary antibodies (1:10000) (Jackson ImmunoResearch, West Grove, PA, USA). The proteins attached to the membrane was measured using enhanced chemiluminescence (ECL) detection system (Santa Cruz Biotechnology, Santa Cruz, CA, USA), according to the manufacturer's instructions.

\section{Gene expression analysis}

Total RNA was isolated using Trizol (TaKaRa Bio, Otsu, Japan) according to the manufacturer's protocols. The concentration of extracted RNA was measured with Nanodrop 2.0 (Thermo scientific, PA, USA) and converted into cDNA with a reverse transcription kit (Invitrogen, Carlsbad, CA, USA). The RT-PCR reaction was composed of 22-40 cycles of denaturation, annealing and extension using Taq polymerase (Kapa Biosystems, Woburn, MA, USA). The primer sequences are as follows Table 1. The reacted products were run on SYBR green stained agarose gel (Invitrogen, Carlsbad, CA, USA). The gel was photographed using gel documentation system (NoBI, Neo science, Seoul, Korea) and determined using ImageJ software (Image J1, National Institutes of Health, Bethesda, MD, USA).

\section{In vivo model of osteoporosis and serum analysis}

Forty female Sprague-Dawley (SD) rats (240-250 g) were provided by Nara Biotech (Seoul, Korea). All experiments were conducted according to the principles of the Institutional Animal Care and The protocol was approved by Committee of the Kyung Hee University Laboratory Animal Center (permission number: KHUASP (SE)-13-051). The rats were acclimatized in the laboratory environment for one week and then they were either sham-operated $(n=8)$ or ovariectomized $(n=32)$. To induce postmenopausal osteoporosis model. The ovariectomized (OVX) group removed both ovaries. In addition, the sham-operated group did not remove the ovaries after laparotomy to give the same stress. Rats were randomly placed into 5 groups $(\mathrm{n}=8)$; (1) Sham, sham-operated rats and distilled water-orally administered; (2) OVX, OVX control rats and distilled water-orally administered; (3) $\mathrm{E}_{2}$, OVX and $17 \beta$-estradiol $(100 \mu \mathrm{g} / \mathrm{kg})$-orally administered; (4) CR-L, OVX and CR $36 \mathrm{mg} / \mathrm{kg}$-orally administered; (5) CR-H, OVX and CR $360 \mathrm{mg} / \mathrm{kg}$-orally administered. Oral administration was carried out every morning for 8 weeks. At the end of the treatment, rats were injected intraperitoneally with a high concentration of pentobarbital sodium $(80 \mathrm{mg} / \mathrm{kg})$ for anesthesia and then blood close to the lethal dose was collected with a cardiac puncture and cervical dislocation was progressed. The uterus and femurs and tibias were collected and weighed. The serum samples were prepared by centrifugation of the collected blood samples (2000 rpm for $10 \mathrm{~min}$ at $4{ }^{\circ} \mathrm{C}$ ) and then stored at $-80^{\circ} \mathrm{C}$. Osteocalcin was measured by Mouse Osteocalcin ELISA Kit (LSBio, WA, USA). Measuring of the TRAP activity was performed as previously described [9].

\section{Histological examination}

The left femur was fixed in $10 \%$ neutral buffered formalin (NBF) for 2 days, demineralized using $10 \%$ Ethylenediamine tetraacetic acid (EDTA-2Na) for 3

Table 1 Primer sequence for RT-PCR analysis

\begin{tabular}{|c|c|c|c|c|c|}
\hline Target genes & Primer sequence & Accession number & Annealing & Cycle & Base pair \\
\hline TRAP & $\begin{array}{l}\text { F: 5'-act tcc cca gcc ctt act acc g-3' } \\
\text { R: 5'-tca gca cat agc cca cac cg-3' }\end{array}$ & NM_007388.3 & $58^{\circ} \mathrm{C}$ & 30 & 381 \\
\hline NFATC1 & $\begin{array}{l}\text { F: } 5^{\prime}-\text { tgc tcc tcc tcc tgc tgc tc-3' } \\
\text { R: } 5^{\prime}-\text { cgt ctt cca cct cca cgt cg-3' }\end{array}$ & NM_198429.2 & $58^{\circ} \mathrm{C}$ & 32 & 480 \\
\hline c-Fos & $\begin{array}{l}\text { F: 5'-atg ggc tct cct gtc aac ac-3' } \\
\text { R: 5'-ggc tgc caa aat aaa ctc ca-3' }\end{array}$ & NM_010234.3 & $58^{\circ} \mathrm{C}$ & 33 & 480 \\
\hline RANK & $\begin{array}{l}\text { F: 5'-aaa cct tgg acc aac tgc ac-3' } \\
\text { R: 5'-acc atc ttc tcc tcc cga gt-3' }\end{array}$ & NM_009399.3 & $53^{\circ} \mathrm{C}$ & 32 & 377 \\
\hline CA2 & $\begin{array}{l}\text { F: } 5^{\prime} \text {-ctc tca gga caa tgc agt gct ga-3' } \\
\text { R: 5'-atc cag gtc aca cat tcc agc a-3' }\end{array}$ & NM_001357334.1 & $58^{\circ} \mathrm{C}$ & 32 & 411 \\
\hline CTK & $\begin{array}{l}\text { F: 5'-agg cgg cta tat gac cac tg-3' } \\
\text { R: 5'-ccg agc caa gag agc ata tc-3' }\end{array}$ & NM_007802.4 & $58^{\circ} \mathrm{C}$ & 27 & 403 \\
\hline MMP-9 & $\begin{array}{l}\text { F: } 5^{\prime} \text {-cga ctt ttg tgg tct tcc cc-3' } \\
\text { R: } 5^{\prime} \text {-tga agg ttt gga atc gac cc-3' }\end{array}$ & NM_013599.4 & $58^{\circ} \mathrm{C}$ & 33 & 258 \\
\hline CTR & $\begin{array}{l}\text { F: 5'-tgc att ccc ggg ata cac ag-3' } \\
\text { R: 5'-agg aac gca gac ttc act gg-3' }\end{array}$ & NM_001355192.1 & $59^{\circ} \mathrm{C}$ & 40 & 393 \\
\hline GAPDH & $\begin{array}{l}\text { F: 5'-act ttg tca agc tca ttt cc-3' } \\
\text { R: 5'-tgc agc gaa ctt tat tga tq-3' }\end{array}$ & NM_008084.3 & $58^{\circ} \mathrm{C}$ & 30 & 267 \\
\hline
\end{tabular}


weeks, and then dehydrated with ethanol, clarified with xylene, and embedded with paraffin. Paraffin embedded tissue was sectioned on a rotary microtome (ZEISS, Oberkochen, GERMANY). The sectioned tissues were stained with hematoxylin-eosin (H\&E). Moreover, to confirm osteoclastogenesis inhibition, TRAP staining proceeded. TRAP-stained tissues were counterstained with methyl green. The histologic changes of the femur caused by the ovariectomy were observed with a light microscope (DP73, Olympus, Tokyo, Japan) (40, 100x).

\section{Immunohistochemical (IHC) staining}

The left femur was fixed in 10\% NBF for 2 days, demineralized using 10\% EDTA-2Na for 3 weeks, and then dehydrated with ethanol, clarified with xylene, and embedded with paraffin. Paraffin embedded tissue was sectioned on a rotary microtome. Endogenous peroxidase was blocked in $3 \% \mathrm{H}_{2} \mathrm{O}_{2} / \mathrm{Mt}-\mathrm{OH}$ for 15 min at room temperature. Then, $20 \mu \mathrm{g} / \mathrm{ml}$ proteinase $\mathrm{K}$ (Thermo fisher, PA, USA) was used for epitope retrieval for $10 \mathrm{~min}$ at $37^{\circ} \mathrm{C}$, for blocking, normal serum (Gibco, Gaithersburg, MD, USA) was reacted at room temperature for $30 \mathrm{~min}$. The tissues were incubated with primary antibody diluted in $0.5 \%$ BSA, including anti-NFATc1 (1:100, Santa Cruz Biotechnology, CA, USA), anti-c-fos (1:100, Santa Cruz Biotechnology, CA, USA) and anti-cathepsin K (1:100, Santa Cruz Biotechnology, CA, USA), at $4{ }^{\circ} \mathrm{C}$ overnight. The tissues were then stored in 1:100 biotinylated secondary antibody (Vector Labs, Burlingame, CA, USA) for 60 min at room temperature. The tissues were incubated in horseradish peroxidase-streptavidin using $\mathrm{ABC}$ kit (Vector Labs, Burlingame, CA, USA) for $30 \mathrm{~min}$ at room temperature and stained with 3,3'-Diaminobenzidine solution (Vector Labs). The tissues were counterstained with hematoxylin, dehydrated and mounted. The immunohistochemical stained tissues were observed with a light microscope $(100,200 \times)$.

\section{Statistical analysis}

All data are presented as mean $\pm \mathrm{S}$. E from at least three or more experiments. Data were evaluated by One-way analysis of variance (ANOVA) between two mean values, and this was followed by Dunnett's multiple comparison test. $P<0.05$ was considered statistically significant.

\section{Results}

\section{Quality assessment of the CR extract}

Chlorogenic acid is a standard marker for the authentication of CR [30]. The chromatogram of the water extract from CR showed many peaks at a retention time of 0 and $30 \mathrm{~min}$, and chlorogenic acid was found at the same retention time as the standards (Fig. 1).

\section{Effects of $C R$ on cell viability}

Before performing the in vitro tests, we determined the cell viability of $\mathrm{CR}$ on osteoclast precursors. All concentrations of $\mathrm{CR}$ were shown to have a viability equivalent

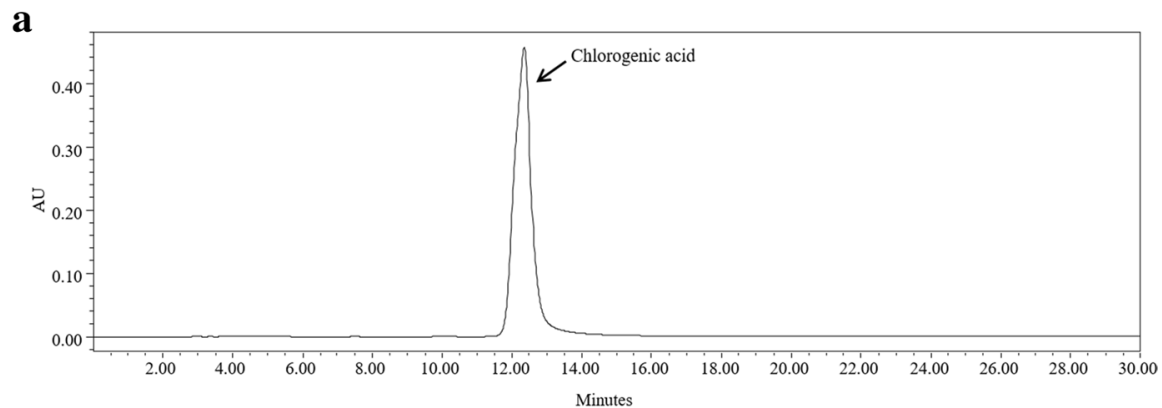

b

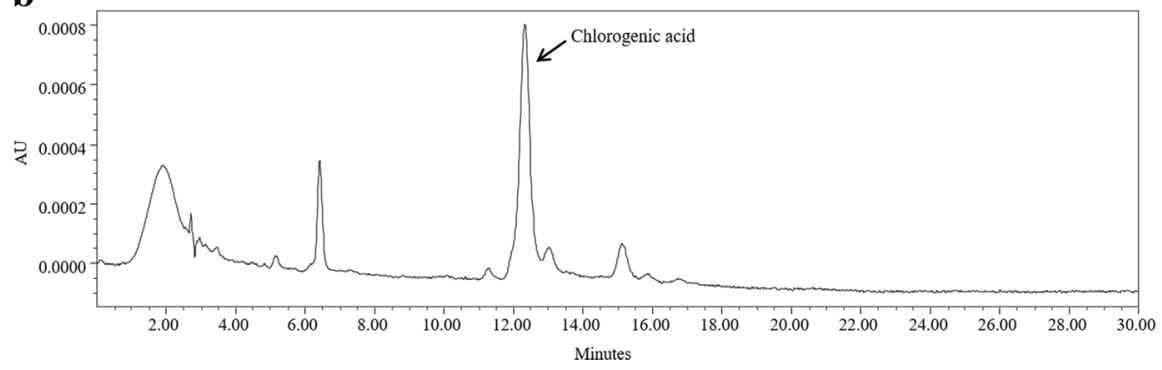

Fig. 1 HPLC Chromatograms of the $\mathbf{a}$ Chlorogenic acid and $\mathbf{b}$ CR 
to the normal. CR did not show any cytotoxicity in the RAW 264.7 cells (Fig. 2).

\section{CR inhibited osteoclastogenesis in RAW 264.7 cells}

To measure the effect of $\mathrm{CR}$ on osteoclast formation using the murine monocyte/macrophage cell line RAW 264.7, RANKL (100 ng/ml) was used to induce TRAPpositive multinucleated osteoclast differentiation in RAW 264.7 cells. CR had inhibitory effects on TRAPpositive cells in a dose-dependent manner (Fig. 3a and b). Furthermore, CR also had an inhibitory effect on the TRAP activity (Fig. 3c). These data are consistent with the inhibitory effects on osteoclast formation.

\section{CR inhibited the bone resorptive activity}

To determine the effect of $\mathrm{CR}$ on the bone resorptive activity, which is a major function of osteoclasts, RAW 264.7 cells were cultured in an osteo strip well plate. CR had an inhibitory effect on bone resorption (Fig. 4a). The measured area was also significantly reduced in the CR treat group (Fig. 4b). These data show that it has an inhibitory effect on the bone resorptive activity.

\section{CR inhibited NFATC1 and c-Fos protein expression}

The effect of $\mathrm{CR}$ on essential osteoclast differentiation indicators, which are NFATc1 and c-Fos, was investigated. NFATc1 is controlled by c-Fos as a master transcription factor for osteoclast differentiation. CR had significant inhibitory effects on NFATc1 protein expression (Fig. 5a); CR also significantly inhibited the c-Fos protein expression (Fig. 5b).

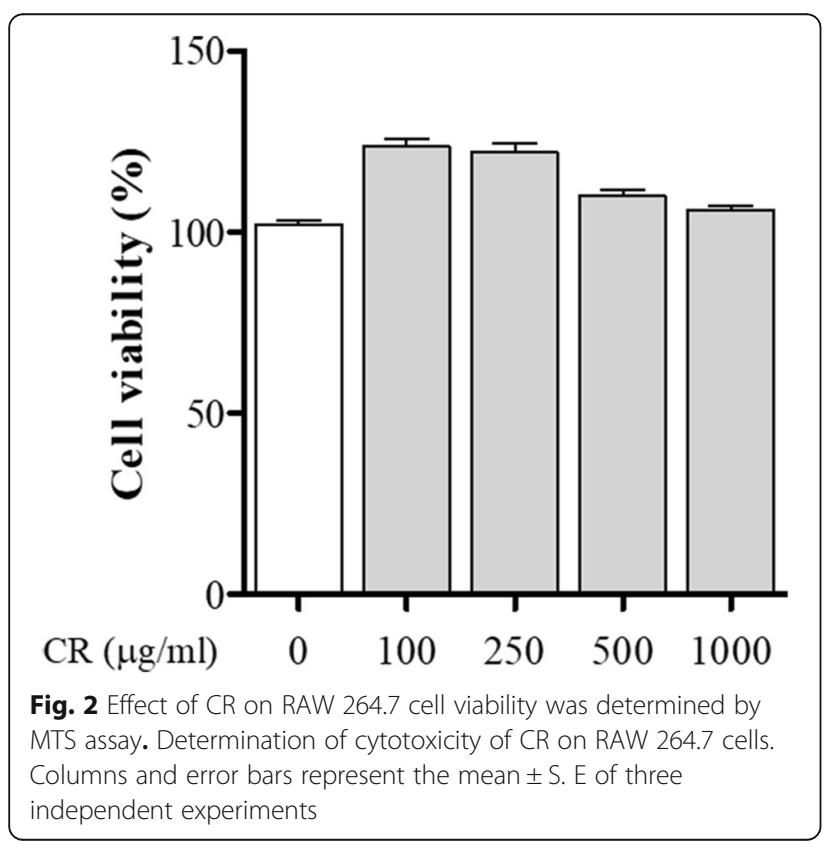

\section{CR inhibited osteoclastogenesis-related genes}

Next, the effect of CR on the mRNA expression of c-Fos and NFATc1 was explored. We also looked at the effect of CR on the activation of osteoclastogenesis marker by RANKL, such as RANK, TRAP, CTK, CTR, MMP-9, and carbonic anhydrase 2 (CA2). CR inhibited the expression of all osteoclastogenesis-related genes by RANKL in a dose-dependent manner (Fig. 6a). In particular, TRAP, c-Fos, CA2, and CTR were effectively inhibited at low concentrations of CR.

\section{CR increased the femur weight in the OVX rat model}

We investigated whether $C R$ suppresses bone loss of OVX rats. After OVX, the OVX group weighed more than the sham group, and the $\mathrm{E}_{2}$ group prevented weight gain via OVX. However, CR groups did not affect (Fig. 7a). Uterine weight decreased through OVX, $E_{2}$ group suppressed uterine weight loss, but $\mathrm{CR}$ groups showed no changes (Fig. 7b). There was no significant change in the femur weight loss in the $\mathrm{E}_{2}$. However, the CR-H group significantly inhibited OVX-induced weight loss of the femur (Fig. 7c).

\section{CR reduced the serum level of osteocalcin and TRAP activity}

To measure the effect of CR treatments on bone turnover markers in OVX rats, we analyzed level of osteocalcin and TRAP in serum. Level of osteocalcin increased by OVX. CR and $E_{2}$ groups had significant inhibitory effects on osteocalcin expression. In particular, the CR-H group had a strong inhibitory effect on osteocalcin (Fig. $7 d)$. As a result of measuring TRAP activity in serum, CR treated group showed suppressive effect (Fig. 7e).

\section{CR had an inhibitory effect on trabecular loss and osteoclastogenesis in the histologically stained femur}

To investigate the effect of CR on bone loss and the number of osteoclasts, we did histological staining of the femurs using H\&E and TRAP staining. As shown in Fig. 8a, OVX reduced the trabecular area of the femoral bone. Compared to the sham group, the difference is significant and $\mathrm{CR}-\mathrm{H}$ and $\mathrm{E}_{2}$ groups significantly inhibit trabecular area reduction (Fig. 8b). Additionally, the OVX group had a significantly increased number of osteoclasts compared with the sham group. The $E_{2}$ and CR-High groups had a significantly decreased number of osteoclasts compared with the OVX group (Fig. 8c). These data consistently show the inhibitory effects of $\mathrm{CR}$ on osteoporosis in the OVX-rat model through the inhibition of osteoclast differentiation. 


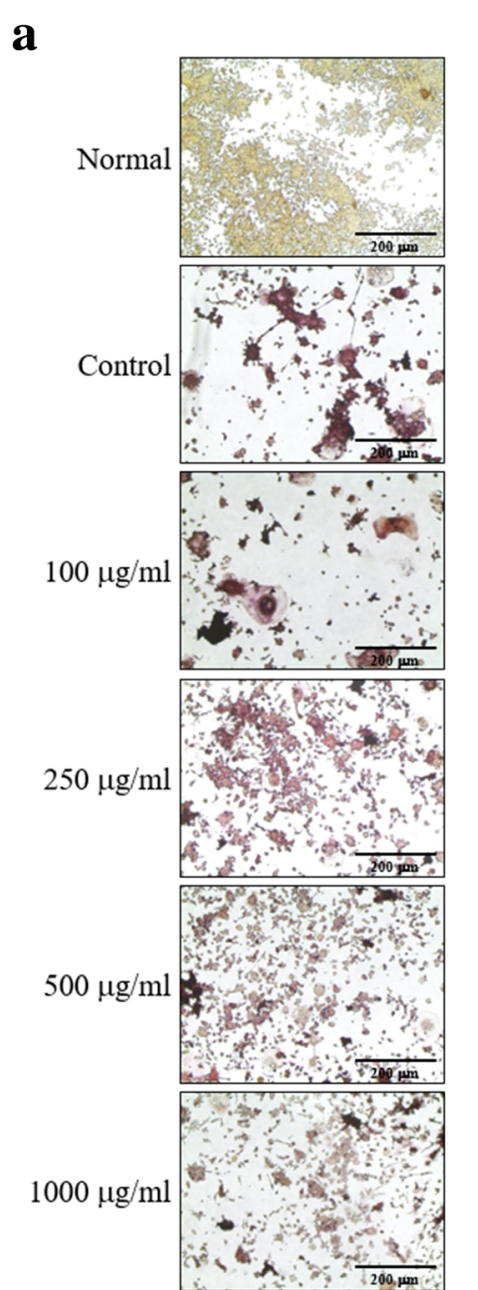

b

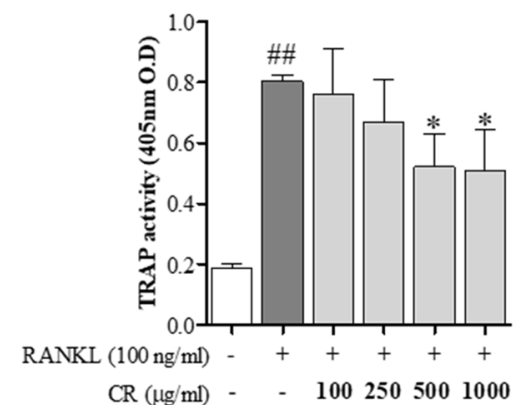

c

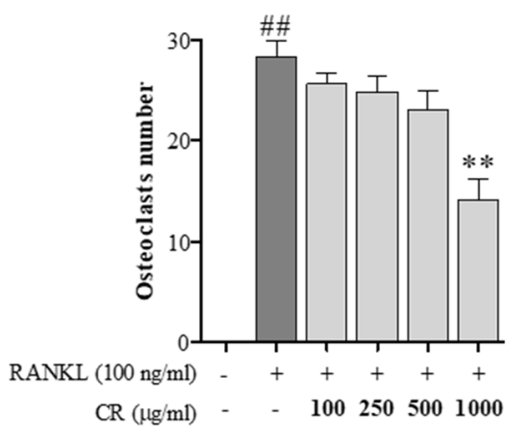

Fig. 3 Effect of CR on osteoclast differentiation. The cells were stained with the TRAP assay kit, and media were collected for the TRAP activity. a TRAPpositive multinuclear cells were captured using an inverted microscope (100x, Scale bars: $200 \mu \mathrm{m})$. b Cells were counted, and $\mathbf{c}$ the media were measured for TRAP activity by an ELISA reader. Columns and error bars represent the mean $\pm S$. E of three independent experiments. ${ }^{\# \#} p<0.01$ compared with normal and ${ }^{* *} p<0.01,{ }^{*} p<0.05$ compared with the control

CR had an inhibitory effect on the expression of NFATc1, c-fos and CTK expression in femur

To examine the effect of $\mathrm{CR}$ on the expression of NFATc1, c-fos and CTK in the femur, IHC staining was performed. As shown in Fig. 9, compared to the sham group, the expression of NFATc1, c-Fos and CTK increased in the OVX group. CR-L and CR-H groups inhibited expression of NFATc1, c-Fos and CTK in the osteoporotic femur. These results suggest that the antiosteoporotic effect of CR is due to the suppression of NFATc1, c-Fos and CTK expression in the femur.

\section{Discussion}

In the present study, we demonstrated that CR is a potent inhibitor of osteoclast differentiation in RAW 264.7 cells by the suppression of important transcription marker.
Moreover, CR inhibited bone loss and osteoclast differentiation in the OVX rat models. The key causal factor of osteoporosis is abnormal bone resorption of osteoclasts. The inhibition of osteoclast differentiation would be a significant treatment strategy for osteoporosis.

It is important that TRAP staining and activity assays are used when identifying the osteoclast phenotype. To examine the effects of $\mathrm{CR}$ on the osteoclast differentiation, RANKL-induced models were used in RAW 264.7 cells [31]. In the present study, CR inhibited osteoclastogenesis and its activity. Osteoclasts are large multinucleated cells with the potential to form resorption lacunae on the bone. Functionally, the pit formation assay is required when identifying the bone resorption activity of osteoclast and the mRNA expression of TRAP, CTK, MMP-9, and CA2 is also 


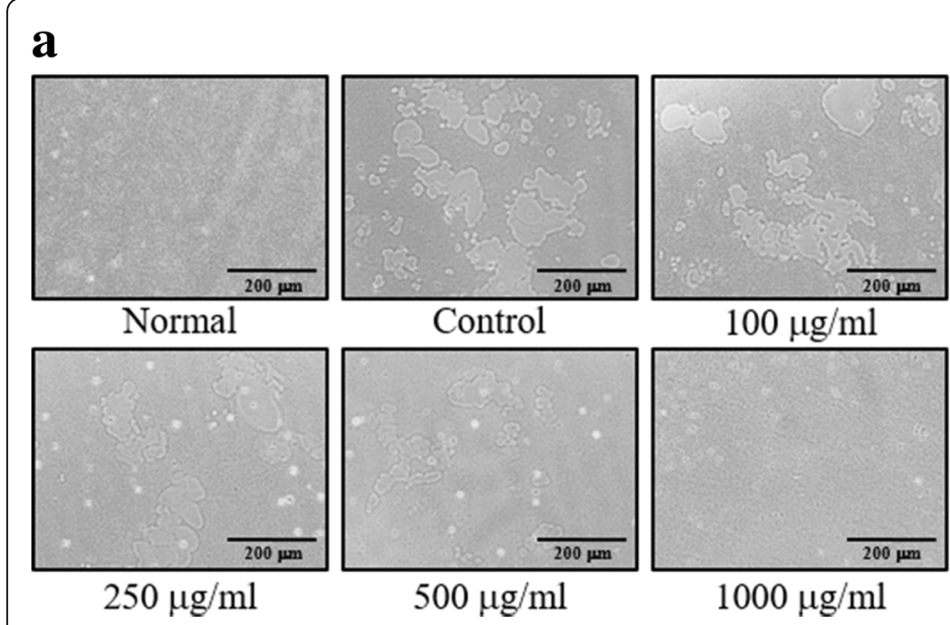

b

Fig. 4 Effect of CR on pit formation. a Pit area was observed under an inverted microscope (100x, Scale bars: $200 \mu m$ ), and $\mathbf{b}$ the Pit area was measured using the ImageJ software. Columns and error bars represent the mean \pm S. E of three independent experiments. ${ }^{\# \#} p<0.01$ compared with normal and ${ }^{* *} p<0.01,{ }^{*} p<0.05$ compared with the control

important because these genes are involved in the bone resorption [13, 32-34]. To confirm the effects of $\mathrm{CR}$ on bone resorption, the bone resorption-related genes were measured by RT-PCR. We found that CR inhibited the pit formation and reduced the RANKLinduced production such as TRAP, CTK, MMP-9, and $\mathrm{CA} 2$ genes. These results suggest that $\mathrm{CR}$ has an inhibitory effect on bone resorption, which is the main function of osteoclasts.

Previously, many studies have confirmed that NFATc1 is the transcriptional factor involved in T-cell maturation, and it has been reported recently to be the master switch regulator for osteoclast formation and function $[5,15,35]$. Other studies have found that even in the absence of RANKL, overexpression of NFATc1 induces osteoclast precursor cells differentiate into osteoclasts. [15]. In addition, NFATc1 regulate various phenotype genes involved in osteoclastogenetsis and bone resorption such as TRAP, CTK, MMP-9, and CTR $[13,15]$. In this study, CR inhibited the mRNA and protein expressions of NFATc1. These data indicate that CR suppresses the mRNA expression of osteoclast-related

\section{$\mathbf{a}$}

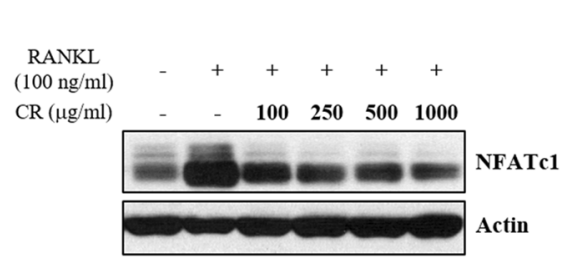

c

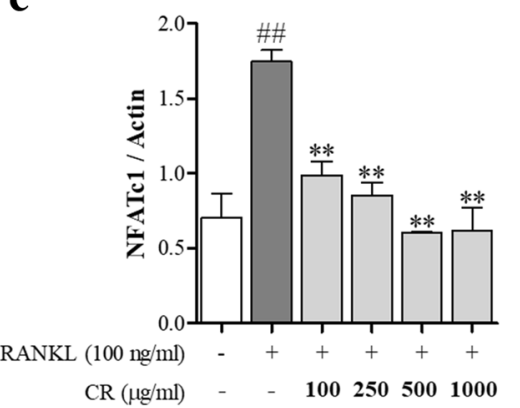

b

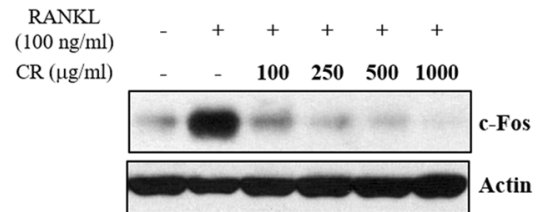

d

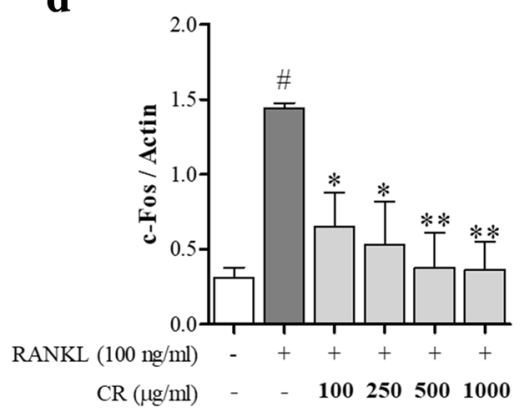

Fig. 5 Effect of CR on the activation of NFATC1 and c-Fos by RANKL. a NFATC1 and $\mathbf{b}$ c-Fos protein expressions were determined by western blot. Expressions of $\mathbf{c}$ NFATc1 and $\mathbf{d}$ c-Fos were normalized to actin. Columns and error bars represent the mean $\pm \mathrm{S}$. E of three independent experiments. ${ }^{\#} p<0.01,{ }^{\#} p<0.05$ compared with normal and ${ }^{* *} p<0.01,{ }^{*} p<0.05$ compared with the control 


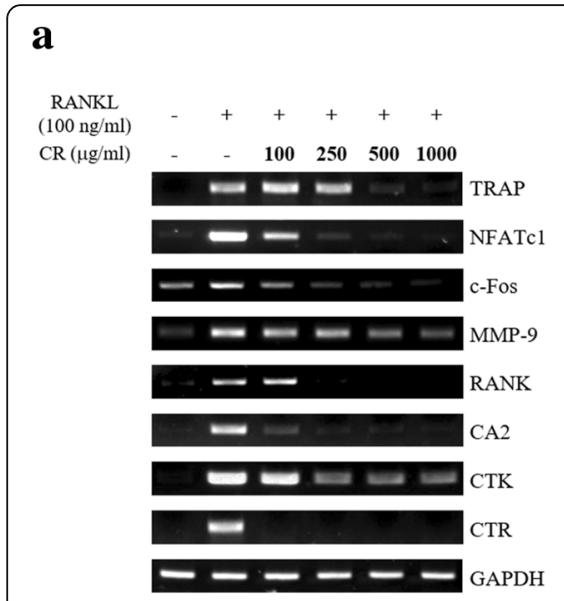

markers through the inhibition of NFATc1. Recently, it has been reported that c-Fos is a key regulator of osteoclastogenesis and bone remodeling [12]. The removal of the gene encoding c-Fos causes defective osteoclast differentiation and osteopetrosis [36]. Whereas the overexpression of c-Fos in osteoclast progenitors improves osteoclastogenesis [37]. In the present study, CR inhibited the mRNA and protein expressions of c-Fos. These results indicate that CR inhibits both NFATc1 and c-Fos, the key factors of this mechanism, and suppresses the differentiation into osteoclasts. c-Fos also regulates osteoclastogenesis-related genes, such as CA2. The promoter of the gene

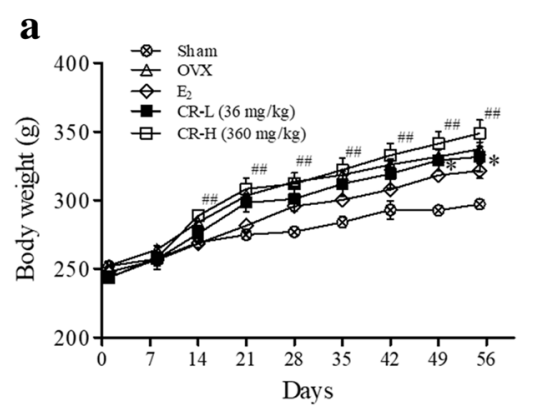

c

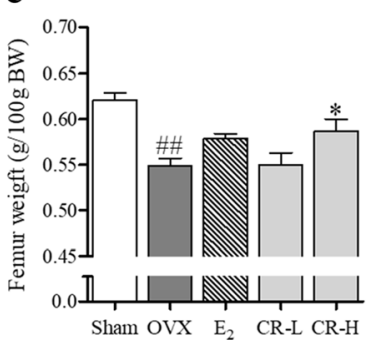

d

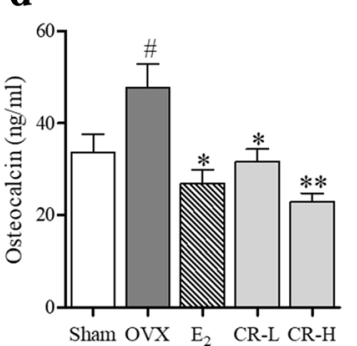

b

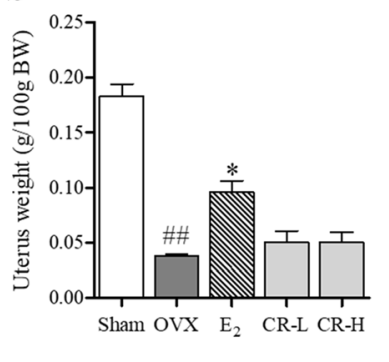

e

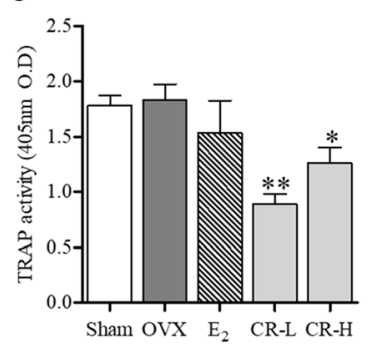

Fig. 7 Effect of CR on the OVX-induced osteoporosis rat-model. a Weekly body weight of OVX-induced SD-rats, $\mathbf{b}$ the rats were sacrificed after weighing the uterus and $\mathbf{c}$ tibia. $\mathbf{d}$ Osteocalcin and $\mathbf{e}$ TRAP activity on serum were measured. Columns and error bars represent the mean $\pm \mathrm{S}$. $\mathrm{E}$ of eight independent experiments. ${ }^{\# \#} p<0.01$ compared with the Sham and ${ }^{*} p<0.05$ compared with OVX 


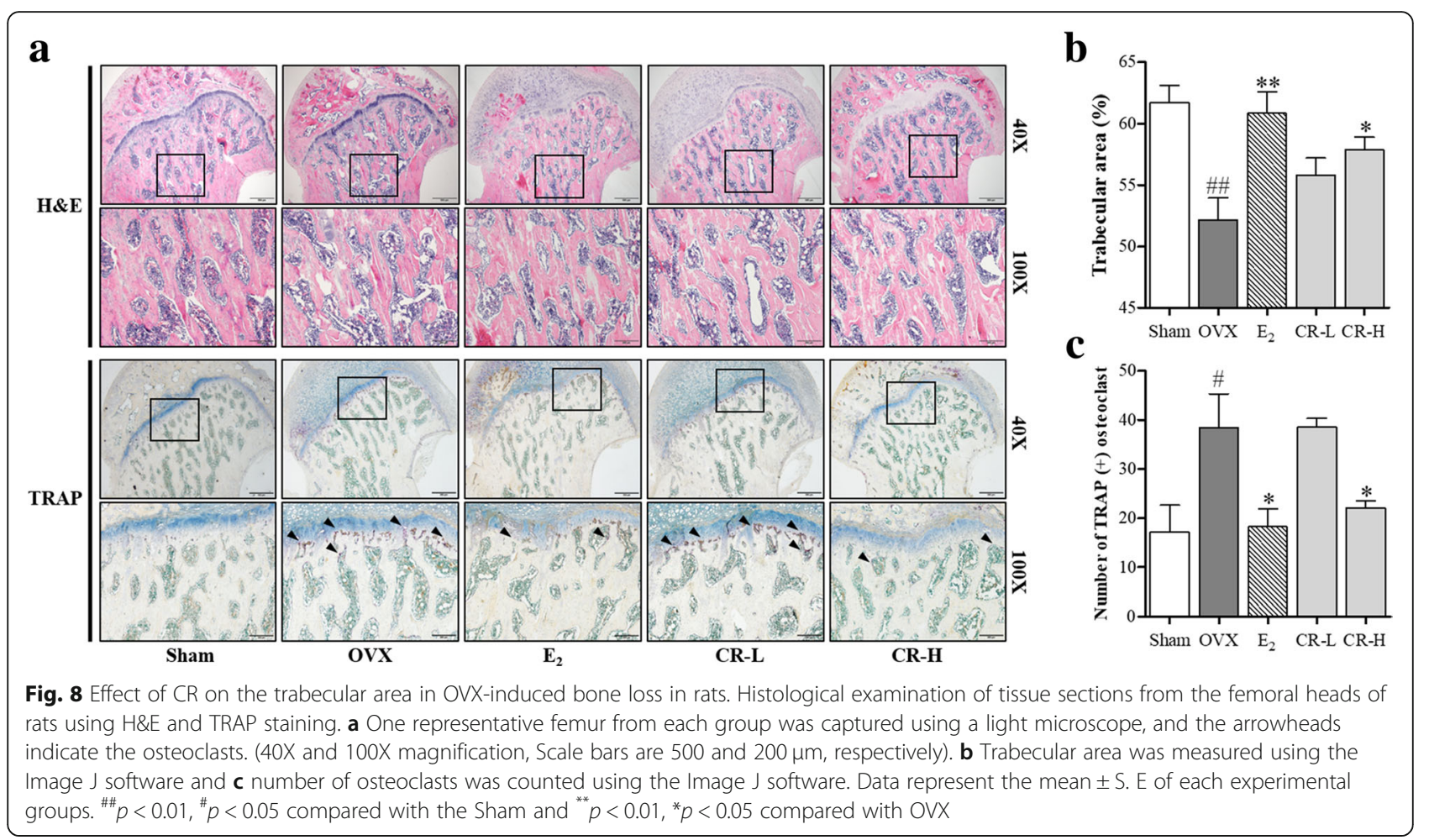

that encodes CA2 is directly regulated by c-Fos overexpression [34]. CA2 is located on the bone surface and acidifies the surface. After that, bone resorption markers lead to absorb [9, 13, 34, 36]. In this study, CR suppresses CA2 through the inhibition of c-Fos. In addition, we also confirmed the expression of RANK, an early mechanism of RANKL-induced osteoclast [5]. CR also inhibited RANK, these results indicate that the effect of CR to inhibit osteoclast differentiation is a result of down-regulation of RANK on the surface of osteoclast precursor cells (Additional file 1).

Bilateral OVX is a generally used experimental method to recapitulate bone remodeling disorders in animal models [38]. As a result of OVX, the increasing osteoclast activity and osteoclastogenesis is the main mechanism reported to result in bone loss in these models [39]. Lack of estrogen through OVX is caused by a unique atrophy of the uterus [40]. In this study, uterine weight decreased after OVX, it is evidence of the success of OVX and supports the results of other research [41, 42]. CR treatment resulted in no changes in uterus weight compared with the OVX group. Moreover, another characteristic of OVX is an increased body weight; CR had no effect on body weight. The mechanism of OVX and body weight is unclear; however, it is expected to be associated with estrogen deficiency. In contrast, the $E_{2}$ group showed smaller decreases in uterus weight and smaller increases in body weight. These data show that
CR does not affect hormone function, such as estrogen [43]. In addition, the induced osteoclasts in the bone increase the concentration of TRAP in the serum. Thus, the serum concentration of osteocalcin, a bone turnover marker, also is increased [44, 45]. In this study, CR significantly inhibited both indicators in serum. TRAP activity was slightly increased by OVX, but the difference was not significant. Although we can't accurately account for the small difference in TRAP activity between Sham and OVX, it is assumed that the experiment period is short [46]. However, we founded that the CR group had inhibitory effect of TRAP activity. These results indicate that $\mathrm{CR}$ inhibits the induction of OVX induced osteoporosis.

Our study also showed that CR reduced bone loss in an OVX rat model [47]. The bone histological examination results indicated that OVX increased the bone resorption area and decreased the bone weight [9]. Low bone mass is a major risk factor for fractures, and OVX significantly increased the bone trabecular area [2, 38]. In this study, the CR-High group showed an inhibitory effect with a reduced trabecular area. Additional, CR inhibited osteoclast differentiation and expression of NFATc1, c-Fos and CTK in femoral tissue. These data indicate that CR is a beneficial treatment for postmenopausal osteoporosis by through inhibition of osteoclast differentiation through inhibition of NFATc1. 


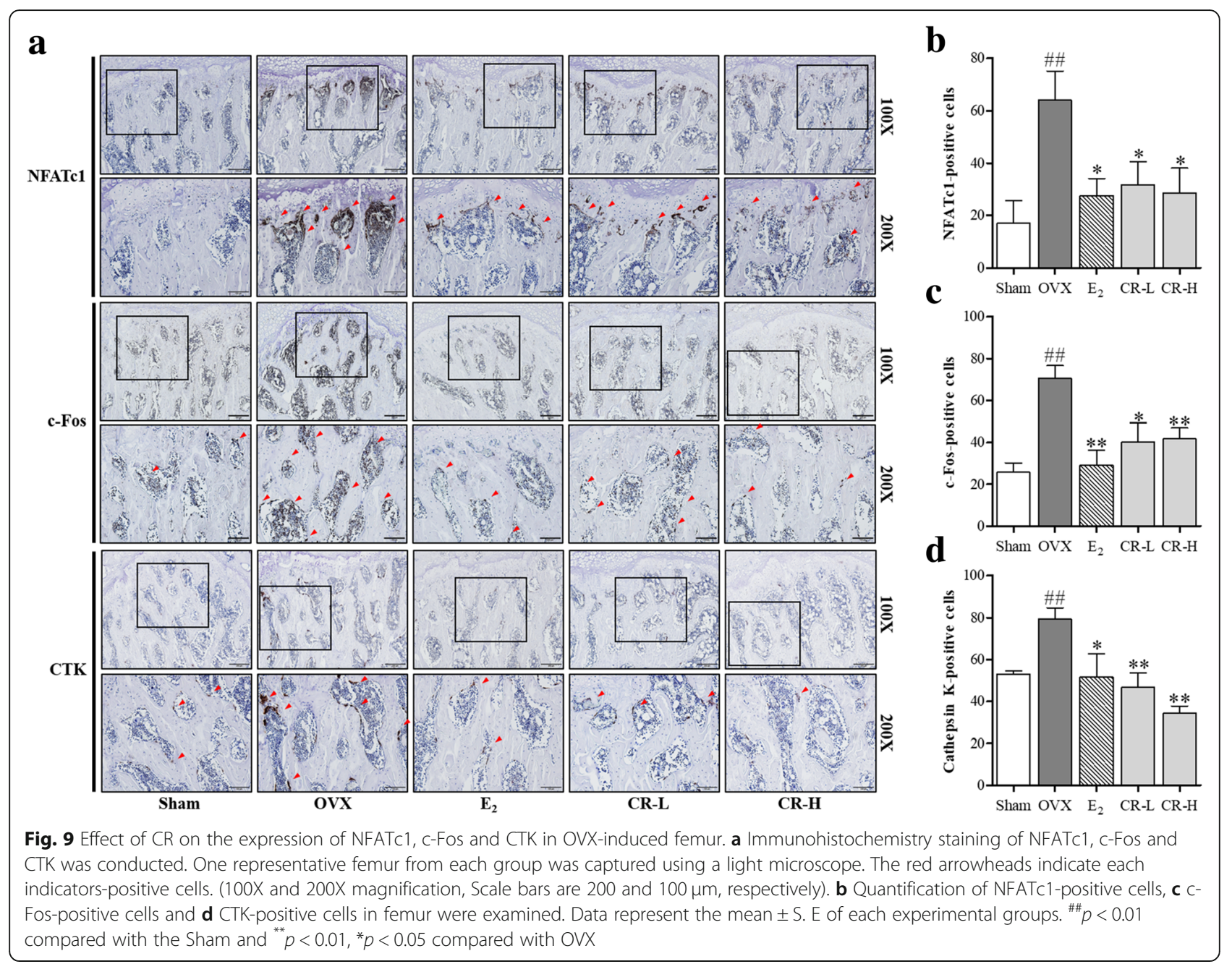

\section{Conclusion}

In conclusion, our findings clearly show that $\mathrm{CR}$ has an inhibitory effect on osteoclastogenesis by RANKL. CR inhibited the expression of osteoclastogenesis markers, such as TRAP, CTK, CTR, MMP-9, RANK, CA2, NFATc1 and c-Fos. Moreover, CR also decreased the OVX-induced bone loss and expression of NFATc1 and CTK in femur. Thus, our results indicate that CR may have potential of a therapeutic herb for bone diseases associated with abnormal osteoclast formation and bone destruction. However, further research is needed, with a particular focus on potential side effects.

\section{Additional file}

Additional file 1: Scheme for CR inhibition of RANKL-induced oateoclas togenesis ( $\vdash, \downarrow$ : inhibition). (TIF $670 \mathrm{~kb}$ ) dehydrogenase; H\&E: Hematoxylin-eosin; HPLC: High performance liquid chromatography; IHC: Immunohistochemistry; MMP-9: Matrix

metallopeptidase-9; NFATc1: Nuclear factor of activated T-cells, cytoplasmic 1; NF-kB: Nuclear factor kappa B; OVX: Ovariectomized; RANK: Receptor activator of the nuclear factor kappa B; RANKL: Receptor activator of the nuclear factor kappa B ligand; RT-PCR: Reverse transcription polymerase chain reaction; TRAF6: Tumor necrosis factor receptor-associated factor 6; TRAP: Tartrate-resistant acid phosphatase

\section{Acknowledgements}

We sincerely appreciate other colleagues in our laboratory for their help and effort in this study.

\section{Authors' contributions}

YS conceptualized the study; KYL and JHK prepared the extract; KYL and JHK performed the in vitro experiments and analyzed the data; JHK and EYK performed the in vivo experiments and analyzed the data; EYK and MY provided technical support; HSJ HPLC experiments and contributed to interpretation of the data. HSJ and YS collected the data, undertook the statistical analyses; KYL and JHK wrote the manuscript; All authors read and agreed on the final manuscript.

\section{Authors' information}

YS and HSJ are a professor in Department of Anatomy, College of Korean Medicine, Kyung Hee University. KYL, JHK and EYK are a graduate student in Department of Anatomy, College of Korean Medicine, Kyung Hee University.
CA2: Carbonic anhydrase 2; CR: Cnidii Rhizoma; CTK: Cathepsin K; CTR: Calcitonin receptor; DMEM: Dulbecco's medium Modified Eagle; ECL: Enhanced chemiluminescence; GAPDH: Glyceraldehyde 3-phosphate 
MY is a research professor in Acupuncture and Meridian Science Research Center, College of Korean Medicine, Kyung Hee University.

\section{Funding}

This work was supported by the National Research Foundation of Korea (NRF) grant funded by the Korea government (MSIT) [grant number: 2017R1A2B4010163]. MSIT had no role in the design and conduct of the study; collection, management, analysis, and interpretation of the data; and preparation, review, or approval of the manuscript.

\section{Availability of data and materials}

All data generated or analysed during this study are included in this published article [and its supplementary information files].

\section{Ethics approval and consent to participate}

The Experimental Animal Facility and protocols were approved by the Institutional Animal Care and Use Committee of Kyung Hee University. (permission number: KHUASP (SE)-13-051). The study does not involve any human data.

\section{Consent for publication}

Not applicable.

\section{Competing interests}

The authors declare that they have no competing interests.

\section{Author details}

'Department of Anatomy, College of Korean Medicine, Kyung Hee University, Seoul 02447, Republic of Korea. ${ }^{2}$ Acupuncture and Meridian Science Research Center, College of Korean Medicine, Kyung Hee University, Seoul 02447, Republic of Korea.

Received: 6 July 2018 Accepted: 23 July 2019

Published online: 09 August 2019

\section{References}

1. Bible JE, Kadakia RJ, Wegner A, Richards JE, Mir HR. One-year mortality after isolated pelvic fractures with posterior ring involvement in elderly patients. Orthopedics. 2013;36(6):760-4.

2. Burge R, Dawson-Hughes B, Solomon DH, Wong JB, King A, Tosteson A. Incidence and economic burden of osteoporosis-related fractures in the United States, 2005-2025. J Bone Miner Res. 2007;22(3):465-75.

3. Teitelbaum SL. Bone resorption by osteoclasts. Science. 2000;289(5484):1504-8.

4. Rodan GA, Martin TJ. Therapeutic approaches to bone diseases. Science. 2000;289(5484):1508-14.

5. Boyle WJ, Simonet WS, Lacey DL. Osteoclast differentiation and activation. Nature. 2003;423(6937):337-42.

6. de Villiers TJ. Bone health and osteoporosis in postmenopausal women. Best Pract Res Clin Obstet Gynaecol. 2009;23(1):73-85.

7. Wensel TM, Iranikhah MM, Wilborn TW. Effects of denosumab on bone mineral density and bone turnover in postmenopausal women. Pharmacotherapy. 2011;31(5):510-23.

8. Jun AY, Kim HJ, Park KK, Son KH, Lee DH, Woo MH, Kim YS, Lee SK, Chung WY. Extract of Magnoliae Flos inhibits ovariectomy-induced osteoporosis by blocking osteoclastogenesis and reducing osteoclast-mediated bone resorption. Fitoterapia. 2012;83(8):1523-31

9. Kim JH, Kim EY, Lee B, Min JH, Song DU, Lim JM, Eom JW, Yeom M, Jung HS, Sohn Y. The effects of Lycii Radicis cortex on RANKL-induced osteoclast differentiation and activation in RAW 264.7 cells. Int J Mol Med. 2016;37(3):649-58.

10. Collin-Osdoby P, Osdoby P. RANKL-mediated osteoclast formation from murine RAW 264.7 cells. Methods Mol Biol. 2012;816:187-202.

11. Suda T, Takahashi N, Udagawa N, Jimi E, Gillespie MT, Martin TJ. Modulation of osteoclast differentiation and function by the new members of the tumor necrosis factor receptor and ligand families. Endocr Rev. 1999:20(3):345-57.

12. Grigoriadis $A E$, Wang $Z Q$, Cecchini MG, Hofstetter W, Felix R, Fleisch $H A$, Wagner EF. C-Fos: a key regulator of osteoclast-macrophage lineage determination and bone remodeling. Science. 1994;266(5184):443-8.

13. Fujisaki K, Tanabe N, Suzuki N, Kawato T, Takeichi O, Tsuzukibashi O, Makimura M, Ito K, Maeno M. Receptor activator of NF-kappaB ligand induces the expression of carbonic anhydrase II, cathepsin K, and matrix metalloproteinase-9 in osteoclast precursor RAW264.7 cells. Life Sci. 2007;80(14):1311-8.

14. Choi HJ, Park YR, Nepal M, Choi BY, Cho NP, Choi SH, Heo SR, Kim HS, Yang MS, Soh Y. Inhibition of osteoclastogenic differentiation by Ikarisoside a in RAW 264.7 cells via JNK and NF-kappaB signaling pathways. Eur J Pharmacol. 2010;636(1-3):28-35.

15. Zhao Q, Wang X, Liu Y, He A, Jia R. NFATc1: functions in osteoclasts. Int J Biochem Cell Biol. 2010;42(5):576-9.

16. Choi HS, Kim MSL, Sawamura M. Constituents of the essential oil of cnidium officinale Makino, a Korean medicinal plant. Flavour Frag J. 2002;17(1):49-53.

17. Gong $X$, Sucher NJ. Stroke therapy in traditional Chinese medicine (TCM): prospects for drug discovery and development. Trends Pharmacol Sci. 1999;20(5):191-6.

18. Bark KM, Heo EP, Han KD, Kim MB, Lee ST, Gil EM, Kim TH. Evaluation of the phototoxic potential of plants used in oriental medicine. J Ethnopharmacol. 2010;127(1):11-8.

19. Kwak DH, Kim JK, Kim JY, Jeong HY, Keum KS, Han SH, Rho YI, Woo WH, Jung KY, Choi BK, et al. Anti-angiogenic activities of Cnidium officinale Makino and Tabanus bovinus. J Ethnopharmacol. 2002; 81(3):373-9.

20. de la Cruz J, Kim DH, Hwang SG. Anti cancer effects of Cnidium officinale Makino extract mediated through apoptosis and cell cycle arrest in the HT29 human colorectal cancer cell line. Asian Pac J Cancer Prev. 2014;15(13): 5117-21.

21. Lee KE, Shin JA, Hong IS, Cho NP, Cho SD. Effect of methanol extracts of Cnidium officinale Makino and Capsella bursa-pastoris on the apoptosis of HSC-2 human oral cancer cells. Exp Ther Med. 2013:5(3):789-92.

22. Jeong JB, Park JH, Lee HK, Ju SY, Hong SC, Lee JR, Chung GY, Lim JH, Jeong $\mathrm{HJ}$. Protective effect of the extracts from Cnidium officinale against oxidative damage induced by hydrogen peroxide via antioxidant effect. Food Chem Toxicol. 2009:47(3):525-9.

23. Lee $\mathrm{SH}$, Lee $\mathrm{JH}$, Oh EY, Kim GY, Choi BT, Kim C, Choi YH. Ethanol extract of Cnidium officinale exhibits anti-inflammatory effects in BV2 microglial cells by suppressing NF-kappaB nuclear translocation and the activation of the PI3K/Akt signaling pathway. Int J Mol Med. 2013;32(4):876-82.

24. Jimi E, Shuto T, Koga T. Macrophage colony-stimulating factor and interleukin-1 alpha maintain the survival of osteoclast-like cells. Endocrinology. 1995;136(2):808-11.

25. Jimi E, Nakamura I, Duong LT, Ikebe T, Takahashi N, Rodan GA, Suda T. Interleukin 1 induces multinucleation and bone-resorbing activity of osteoclasts in the absence of osteoblasts/stromal cells. Exp Cell Res. 1999:247(1):84-93.

26. Zwerina J, Tzima S, Hayer S, Redlich K, Hoffmann O, Hanslik-Schnabel B, Smolen JS, Kollias G, Schett G. Heme oxygenase $1(\mathrm{HO}-1)$ regulates osteoclastogenesis and bone resorption. FASEB J. 2005;19(14):2011-3.

27. Yi T, Leung KS, Lu GH, Zhang H. Comparative analysis of Ligusticum chuanxiong and related umbelliferous medicinal plants by high performance liquid chromatography-electrospray ionization mass spectrometry. Planta Med. 2007;73(4):392-8.

28. Wang JY, Chen WM, Wen CS, Hung SC, Chen PW, Chiu JH. Du-Huo-Jisheng-tang and its active component Ligusticum chuanxiong promote osteogenic differentiation and decrease the aging process of human mesenchymal stem cells. J Ethnopharmacol. 2017;198:64-72.

29. Alexandersen P, Toussaint A, Christiansen C, Devogelaer JP, Roux C, Fechtenbaum J, Gennari C, Reginster JY. Ipriflavone multicenter European fracture S: Ipriflavone in the treatment of postmenopausal osteoporosis: a randomized controlled trial. Jama. 2001:285(11):1482-8.

30. Baek ME, Seong GU, Lee YJ, Won JH. Quantitative analysis for the quality evaluation of active ingredients in Cnidium Rhizome. Yakhak Hoeji. 2016; 60(5):227-34.

31. Hsu H, Lacey DL, Dunstan CR, Solovyev I, Colombero A, Timms E, Tan HL, Elliott G, Kelley MJ, Sarosi I, et al. Tumor necrosis factor receptor family member RANK mediates osteoclast differentiation and activation induced by osteoprotegerin ligand. Proc Natl Acad Sci U S A. 1999; 96(7):3540-5.

32. Halleen JM, Raisanen S, Salo JJ, Reddy SV, Roodman GD, Hentunen TA, Lehenkari PP, Kaija H, Vihko P, Vaananen HK. Intracellular fragmentation of bone resorption products by reactive oxygen species generated by osteoclastic tartrate-resistant acid phosphatase. J Biol Chem. 1999;274(33):22907-10. 
33. Saftig $\mathrm{P}$, Hunziker $\mathrm{E}$, Everts $\mathrm{V}$, Jones $\mathrm{S}$, Boyde A, Wehmeyer $\mathrm{O}$, Suter $\mathrm{A}$, von Figura K. Functions of cathepsin $\mathrm{K}$ in bone resorption. Lessons from cathepsin K deficient mice. Adv Exp Med Biol. 2000;477:293-303.

34. David JP, Rincon M, Neff L, Horne WC, Baron R. Carbonic anhydrase II is an AP-1 target gene in osteoclasts. J Cell Physiol. 2001;188(1):89-97.

35. Takayanagi H. Osteoimmunology: shared mechanisms and crosstalk between the immune and bone systems. Nat Rev Immunol. 2007; 7(4):292-304.

36. Wang ZQ, Ovitt C, Grigoriadis AE, Mohle-Steinlein U, Ruther U, Wagner EF. Bone and haematopoietic defects in mice lacking c-fos. Nature. 1992; 360(6406):741-5.

37. Miyauchi A, Kuroki Y, Fukase M, Fujita T, Chihara K, Shiozawa S. Persistent expression of proto-oncogene $\mathrm{c}$-fos stimulates osteoclast differentiation. Biochem Biophys Res Commun. 1994;205(3):1547-55.

38. Kalu DN. The ovariectomized rat model of postmenopausal bone loss. Bone Miner. 1991;15(3):175-91.

39. Lyritis GP, Georgoulas T, Zafeiris CP. Bone anabolic versus bone anticatabolic treatment of postmenopausal osteoporosis. Ann N Y Acad Sci. 2010;1205:277-83.

40. Versi E, Harvey MA, Cardozo L, Brincat M, Studd JW. Urogenital prolapse and atrophy at menopause: a prevalence study. Int Urogynecol J Pelvic Floor Dysfunct. 2001;12(2):107-10.

41. Kim T, Ha H, Shim KS, Cho WK, Ma JY. The anti-osteoporotic effect of Yijung-tang in an ovariectomized rat model mediated by inhibition of osteoclast differentiation. J Ethnopharmacol. 2013;146(1):83-9.

42. Noorafshan A, Dabbaghmanesh MH, Tanideh N, Koohpeyma F, Rasooli R, Hajihoseini M, Bakhshayeshkaram M, Hosseinabadi OK. Stereological study of the effect of black olive hydroalcoholic extract on osteoporosis in vertebra and tibia in ovariectomized rats. Osteoporos Int. 2015;26(9):2299-307.

43. Choi SY, Park D, Yang G, Lee SH, Bae DK, Hwang SY, Lee PK, Kim YB, Kim IH, Kang HG. Effects of sigma anti-bonding molecule calcium carbonate on bone turnover and calcium balance in ovariectomized rats. Lab Anim Res. 2011;27(4):301-7.

44. Habermann B, Eberhardt C, Feld M, Zichner L, Kurth AA. Tartrate-resistant acid phosphatase 5b (TRAP 5b) as a marker of osteoclast activity in the early phase after cementless total hip replacement. Acta Orthop. 2007;78(2):221-5.

45. Lelovas PP, Xanthos TT, Thoma SE, Lyritis GP, Dontas IA. The laboratory rat as an animal model for osteoporosis research. Comp Med. 2008;58(5):424-30.

46. Tantikanlayaporn D, Wichit P, Weerachayaphorn J, Chairoungdua A, Chuncharunee A, Suksamrarn A, Piyachaturawat P. Bone sparing effect of a novel phytoestrogen diarylheptanoid from Curcuma comosa Roxb. in ovariectomized rats. PloS one. 2013;8(11):e78739.

47. Wronski TJ, Lowry PL, Walsh CC, Ignaszewski LA. Skeletal alterations in ovariectomized rats. Calcif Tissue Int. 1985:37(3):324-8.

\section{Publisher's Note}

Springer Nature remains neutral with regard to jurisdictional claims in published maps and institutional affiliations.

Ready to submit your research? Choose BMC and benefit from:

- fast, convenient online submission

- thorough peer review by experienced researchers in your field

- rapid publication on acceptance

- support for research data, including large and complex data types

- gold Open Access which fosters wider collaboration and increased citations

- maximum visibility for your research: over $100 \mathrm{M}$ website views per year

At $\mathrm{BMC}$, research is always in progress.

Learn more biomedcentral.com/submissions 